\title{
FAIR - from a few-body perspective
}

\author{
T. Aumann ${ }^{1}$, K. Langanke ${ }^{1,2,3}$, K. Peters ${ }^{1,4}$, Th. Stöhlker ${ }^{1,5}$ \\ 1 GSI Helmholtzzentrum für Schwerionenforschung, Darmstadt, Germany \\ 2 Technische Universität Darmstadt, Germany \\ 3 Frankfurt Institute for Advanced Studies, Frankfurt, Germany \\ 4 Goethe-Universität Frankfurt, Germany \\ 5 Ruprecht-Karls Universität Heidelberg, Germany
}

\begin{abstract}
In the next years the Facility for Antiproton and Ion Research FAIR will be constructed at the GSI Helmholtzzentrum für Schwerionenforschung in Darmstadt, Germany. This new accelerator complex will allow for unprecedented and pathbreaking research in hadronic, nuclear, and atomic physics as well as in applied sciences. This manuscript will discuss some of these research opportunities, with a focus on few-body physics.
\end{abstract}

\section{The Facility for Antiproton and Ion Research}

FAIR is the next generation facility for fundamental and applied research with antiproton and ion beams [1]. It will provide worldwide unique accelerator and experimental facilities, allowing for a large variety of unprecedented forefront research in physics and applied sciences. FAIR is an international project with 16 partner countries and more than 2500 scientists and engineers involved in the planning and construction of the accelerators and associated experiments. FAIR will be realized in a stepwise approach. The Modularized Start Version, comprising the Heavy-Ion Synchroton SIS100, the antiproton facilty, the Superconducting Fragment Separator (Super FRS) and experimental areas and novel detectors for atomic, hadron, heavyion, nuclear, and plasma physics and applications in material sciences and biophysics, is expected to be operational in 2016. Completion of FAIR, including the synchroton SIS300, to follow soon after.

The main thrust of FAIR research focuses on the structure and evolution of matter on both a microscopic and on a cosmic scale - bringing our Universe into one laboratory. In particular, FAIR will expand our knowledge in various scientif $\mathrm{c}$ f elds beyond current frontiers addressing:

- the investigation of the properties and the role of the strong (nuclear) force in shaping basic building blocks of the visible world around us and its role in the evolution of the universe;

- tests of symmetries and predictions of the standard model and search for physics beyond in the electroweak sector and in the domain of the strong interaction;

- the propoerties of matter under extreme conditions, both at the subatomic as well as the macroscopic scale of matter;
- applications of high-intensity, high-quality ion and antiproton beams in research areas that provide the basis for, or directly addresses, issues of applied sciences and technology.

The basis for the realization of these wide-spread and pathbreaking research activities are worldwide unique accelerator and experimental facilities (Fig. 1). FAIR will

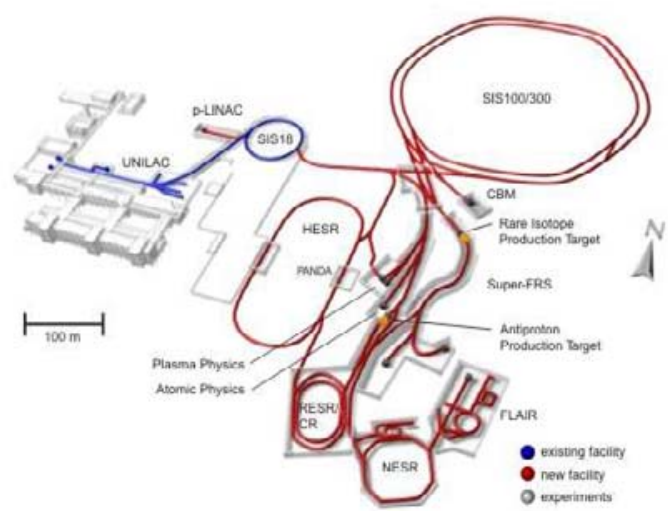

Fig. 1. The Facility for Antiproton and Ion Research.

allow, in comparison to the existing GSI facilities, to increase the beam intensities by factors 100 to 10000 and the beam energies by factors 15-20. Moreover, the use of beam cooling techniques enables the production of antiproton and ion beams of highest quality, i.e. with very precise energy and extremely f ne prof le. Upon completion, the FAIR accelerator complex can support up to 5 experimental programs simultaneously with beams of different ion species in genuine parellel operation. This unique feature is made possible by an optimal balance of the usage of 
the various accumulator, collector and experimental storage rings.

In this manuscript we will discuss some of the outstanding research opportunities at FAIR, concentrating, in the spirit of this conference, on few-body physics. This gives only a restricted glimpse on the research program at FAIR, omitting important aspects. For example,

- the CBM/HADES experiment [2] will explore the phase diagram of Quantumchromodynamics (QCD) in the region of very high baryon densities and moderate temperatures, supplementing related programs at the Relativistic Heavy-Ion Collider and the Large Hadron Collider, which probe the phase diagram at high temperatures and almost zero net baryon densities. The aim of CBM/HADES is to study the nuclear matter equation of state, to search for the predicted $f$ rst order phase transition between hadronic and partonic matter, and to search for the QCD critical point and the chiral phase transition;

- the availability of unprecedentedly intense secondary beams of short-lived nuclei, provided by the Super FRS, opens the unique opportunity to study the evolution of nuclear structure into the yet unexplored territory of the nuclear chart and to determine the properties of many short-lived nuclei which are produced in explosive astrophysical events and crucially inf uence their dynamics and associated nucleosynthesis processes $[3,4]$;

- the PANDA experiment [5] at the high energy storage ring (HESR) will investigate antiproton annihilation reactions to study a broad range of topics dealing with the complex structure of hadrons. High luminosities and high precision will allow unprecedented measurements to understand the binding among quarks. This will be accomplished by studying bound states from light to heavy quarks, in the vacuum and inside hadronic matter, in single mesons and baryons and as constituents in a nucleus.

- for plasma physics the availability of high-energy, highintensity ion-beams enables the investigation of HighEnergy Dense Matter in regimes of temperature, density and pressure, as they exist insides of stars and large planets, but which have not been experimentally accessible so far [6];

- for atomic physics and related felds, the new facility will provide the highest intensities of relativistic beams of both stable and unstable heavy nuclei, in combination with the strongest possible electromagnetic f elds, thus allowing to perform atomic spectroscopy in critical as well as super-critical f elds with utmost accuracy. In combination with novel instrumentation, new f elds will be opened in addition by the strongly enhanced production yields for unstable exotic nuclei, allowing for accurate tests of the standard model.

- the study of the biological effectiveness of high-energy and high-intensity beams is a prerequisite to estimate the expected radiation damage induced by cosmic rays on Moon and Mars missions and to solve related protection issues.
In the following sections we will present highlights of the FAIR research program in few-body aspects in atomic, hadronic and nuclear physics.

\section{Few-body aspects in Nuclear Physics}

\subsection{Three-body Halo nuclei}

Light nuclei far from stability provide a rich testing ground for nuclear-structure effects, there theoretical description and the underlying forces. It is for light nuclei, for which the neutron dripline can be approached experimentally already nowadays. Clustering and few-body aspects play a particular role in this region of the nuclear chart. If the dripline is approached, nuclei might be bound only because of pairing. For the cases of ${ }^{6} \mathrm{He}$ and ${ }^{11} \mathrm{Li}$, for instance, the situation is found that the isotopes with one less neutron are not bound. These so called Borromean nuclei may be described as three-body systems, while none of the two-body subsystems is bound. ${ }^{6} \mathrm{He}$ is the best known example with a clear three-body $\alpha+n+n$ structure, which we will discuss as an example here. The $\alpha$ core is very tightly bound while the $2 n$ separation threshold for ${ }^{6} \mathrm{He}$ is less than $1 \mathrm{MeV}$ giving rise to a rather extended neutron density distribution. Charge- and matter radii have been measured by different experimental techniques supporting the picture of ${ }^{6} \mathrm{He}$ being a $2 n$ Halo nucleus. An interesting observable which is sensitive to the geometrical structure of such a three-body system is the dipole strength associated to the separation of the neutrons from the core. We show in Figure 2 an example of a measurement of the differential electromagnetic excitation cross section, from which the dipole strength distribution can be extracted as shown in this Figure. The experiment has been performed at GSI using a $240 \mathrm{MeV} / \mathrm{u}^{6} \mathrm{He}$ beam [10] impinging on a lead target. The integrated strength in this energy re-



Fig. 2. Dipole-strength distribution in ${ }^{6} \mathrm{He}$. The shaded area ref ects the experimental result obtained from a Coulomb breakup experiment [10], the dashed and dotted curves display results from the three-body models of Refs. [9] and [8], respectively. (Figure from Ref. [10].) 


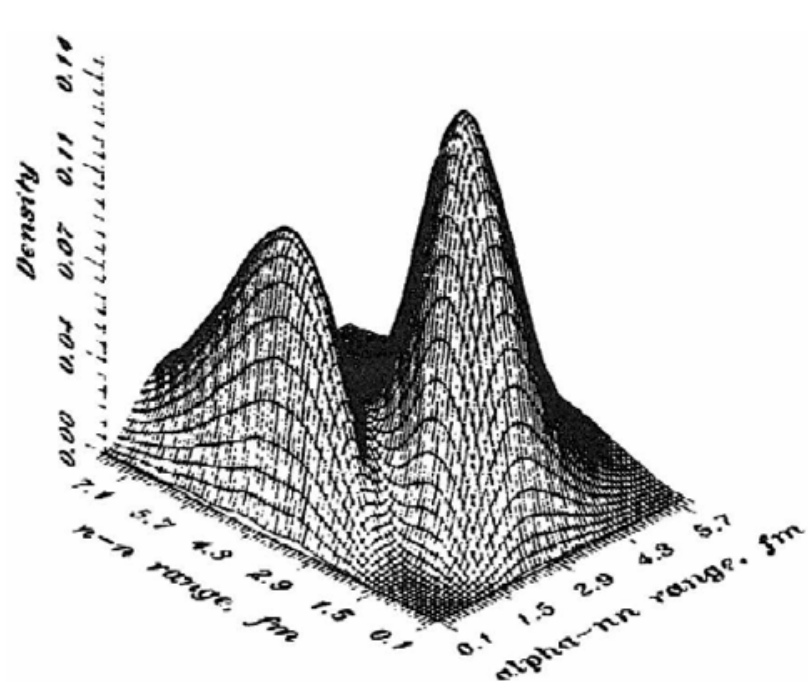

Fig. 3. Density distribution for ${ }^{6} \mathrm{He}$ as calculated in a three-body model [7]

gion exhausts the energy-weighted cluster sum rule, which refers to the relative dipole motion between the core and the halo neutrons. The non-energy weighted sum rule, on the other hand, is related to the distance between the center of mass of the two neutrons and that of the whole nucleus, and is thus sensitive to the spatial distribution including neutron-neutron correlations in the ground state wave function. Figure 3 shows the spatial density distribution for ${ }^{6} \mathrm{He}$ as calculated in a three-body model [7]. One observes clearly two components, one corresponding to a very pronounced 'di-neutron' correlation. From the data, a value $r_{\alpha-2 n}=3.36 \pm 0.29 \mathrm{fm}$ is deduced for the rms distance between the $\alpha$ core and the two neutrons, which is in good agreement with the three-body models, thus supporting the description of ${ }^{6} \mathrm{He}$ by the three-body models including a strong $n-n$ spatial correlation.

\subsection{Few-body nuclear systems beyond the dripline}

In a similar manner, the decay of unbound systems beyond the dripline can be studied by kinematically complete measurements of reactions with radioactive beams. Besides the reconstruction of the relative-energy spectra exhibiting resonance structures in the continuum if present, also angular correlations in the decay of such few-body system may be observed. An example is the super-heavy hydrogen ${ }^{5} \mathrm{H} .{ }^{5} \mathrm{H}$ is unbound and decays into a triton plus two neutrons, constituting again a three-body system. In a high-energy reaction, ${ }^{5} \mathrm{H}$ can be produced for instance in a proton knockout reaction from ${ }^{6} \mathrm{He}$. Observing the three particles in the $\mathrm{f}$ nal channel allows reconstructing the invariant-mass spectrum. A result from an experiment performed at the LAND setup at GSI is shown in Figure 4. A broad peak-like structure is observed at around $3 \mathrm{MeV}$, which is in good agreement with a three-body microscopic calculation [12] assuming a $t+n+n$ structure with a ground-state spin of $I^{\pi}=1 / 2^{+}$. The data have been further analyzed by inspecting the energy- and angular correlations in the three-body

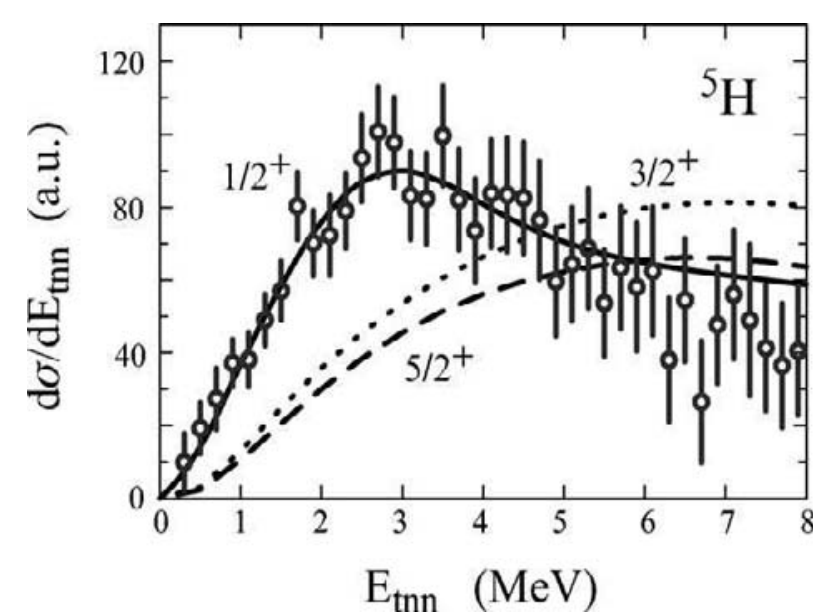

Fig. 4. Invariant-mass spectrum $E_{t n n}$ constructed from the coincident measurement of triton and two neutrons after a one-proton knockout reaction of a $240 \mathrm{MeV} /$ nucleon ${ }^{6} \mathrm{He}$ beam in a carbon target. The curves display microscopic three-body calculations [12] assuming different ground-state spins for ${ }^{5} \mathrm{H}$ as indicated. (From Meister et al. [11].)

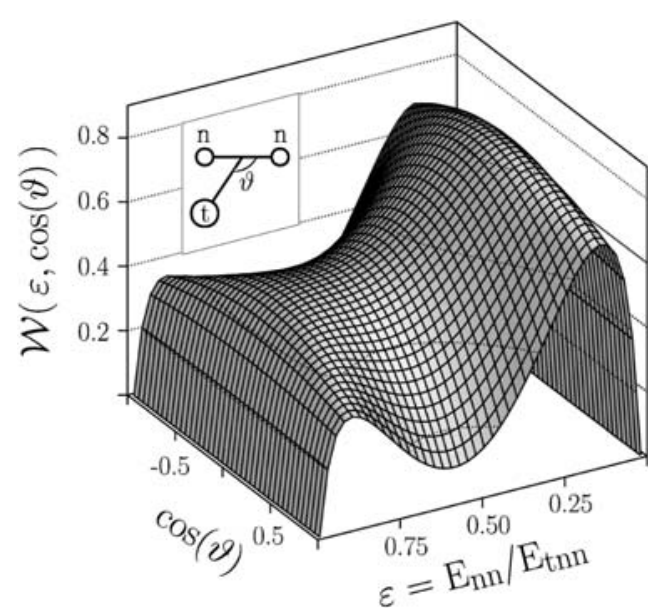

Fig. 5. Probability distribution $\mathcal{W}(\epsilon, \cos (\vartheta))$ with amplitudes resulting from a $\mathrm{ft}$ of a series expansion of the f nal-state wave function to the experimental correlation spectra. The inset sketches the used Jacobi coordinate system. (From Meister et al. [13].)

decay of ${ }^{5} \mathrm{H}$ : The distributions are constructed for two different Jacobi coordinate systems and $\mathrm{ftted}$ by theoretical ones derived from a (truncated) series expansion of the f nal-state wave function into hyperspherical harmonics. From a simultaneous $\mathrm{ft}$ of energy- and angular distributions in both Jacobi coordinate frames, the probability distributions $\mathcal{W}(\epsilon, \cos (\vartheta))$ are obtained [13]. Figure 5 shows the result for one Jacobi system as indicated in the inset. The $n-n$ relative energy $\epsilon=E_{n n} / E_{t n n}$ is given in units of the total available kinetic energy $E_{t n n}$ in the three-body system. The correlation function exhibits two maxima corresponding to the two conf gurations where the neutrons are close together ("di-neutron" conf guration) and far apart from each other ("cigar-like" conf guration), respectively, similar to the ${ }^{6} \mathrm{He}$ case discussed above. The examples 
demonstrate that kinematical complete measurements of reactions with high-energy radioactive beams are a powerful tool to investigate few-body correlations of exotic systems. The study of three-body correlations in the decay of unbound nuclear systems beyond the neutron dripline is particularly useful to obtain information on the character of the usually broad resonances in the continuum. The method has been applied recently to heavy He and Li isotopes beyond the dripline [14,15]. Given the much higher primary-beam intensities expected at the FAIR facility, the investigation of light nuclei at and beyond the neutron dripline can be extended to heavier systems. The next-generation instrumentation as planned for the R3B setup at FAIR will in addition allow for detecting in addition reaction channels with more neutrons in the $\mathrm{f}$ nal state. Multi-neutron droplets formed in reactions of light dripline nuclei is an example.

\subsection{Clustering in Nuclei}

The halo nuclei as discussed above are clustered systems. In particular the $\alpha$ clustering in nuclei has attracted much interest in the past. The quasi-free knockout reaction of nucleons or clusters is an appropriate tool to extract spectroscopic factors. Recently, an experimental scheme has been developed for the kinematically complete measurement of quasi-free scattering of protons in inverse kinematics at high energy, which is applicable for radioactive beams. First experiments have been carried out at the LAND-R3B facility at GSI. The study of nucleon knockout reactions as $(p, 2 p)$ and $(p, p n)$ will give insight into the single-particle structure of nuclei far from stability. In particular, the reduction of single-particle occupancies related to nucleonnucleon correlations will be studied as a function of neutronproton asymmetry. The method can also be used for studying clustering in nuclei by utilizing, e.g., the (p,p $\alpha)$ reaction. Chulkov etal. [16] have analyzed knockout reaction channels from ${ }^{6} \mathrm{He}$ from an experiment, which was optimized for the measurement of elastic $p$ scattering off ${ }^{6} \mathrm{He}$. Recoil protons are detected in the active hydrogen target. Figure 6 shows the angular correlations of recoiliong protons and $\alpha$ particles in the reaction ${ }^{6} \mathrm{He}+p \rightarrow \alpha+p+X$. Two distinct regions are identif ed which are attributed to knockout of a neutron or an $\alpha$ particle from ${ }^{6} \mathrm{He}$, respectively. From this measurement, a spectroscopic factor for the $\alpha$ cluster close to unity has been extracted, as expected for ${ }^{6} \mathrm{He}$, which exhibits a rather clean $\alpha+n+n$ cluster structure as discussed at the beginning of this section. Such experiments are being planned to be performed at the present LAND-R3B setup utilizing a prototype detection scheme for the measurement of quasi-free knockout reactions. This method will exploit its full potential once the R3B experiment at FAIR will go into operation.

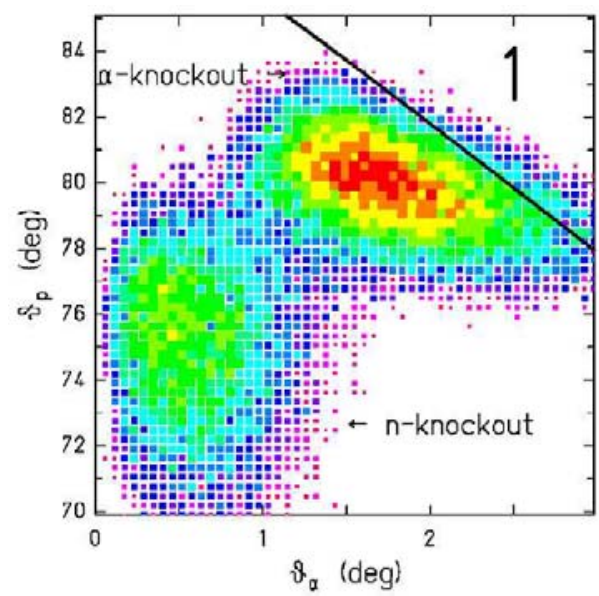

Fig. 6. Correlations between angles of recoil protons and $\alpha$ particles in the ${ }^{1} \mathrm{H}+{ }^{6} \mathrm{He}$ reaction at around $700 \mathrm{MeV} / \mathrm{u}$ [16]. The two distinct regions are associated to quasifree knockout of a neutron or $\alpha$ particle from ${ }^{6} \mathrm{He}$. (From Chulkov et al. [16].)

\section{Few-body aspects in Hadron Physics}

\subsection{QCD bound states}

The study of QCD bound states is of fundamental importance for a better, quantitative understanding of QCD. Particle spectra can be computed within the framework of nonrelativistic potential models, effective f eld theories and Lattice QCD (LQCD). Precision measurements are needed to distinguish between the different approaches and identify the relevant degrees of freedom.

The spectrum of charmonium, like bottomonium, is very similar (apart from the scale) to the spectrum of positronium. It is therefore suggestive to assume that $c \bar{c}$ an $b \bar{b}$ (so called conventional mesons) can be understood in the strong interaction in an analogue way as positronium in electroweak interactions. This would imply a Coulomblike potential and a term which takes care of linear conf nement which is by far not sufficient to explain the spectrum of hadrons. The coherent exchange of gluons which manifest in a gluon tube is an important aspect when one wants to understand the binding among strongly interacting particles. Together with mesic excitations and pure gluonic states which are possible in QCD they are are usually present in the wave function of hadrons and are often referred to as Fock-States of the ground state meson. The Fock-States may decouple from the ground state and thus being individually observable as individual states. Therefore the QCD spectrum is richer than that of the naive quark model (see Fig. 7).

We distinguish conventional, gluonic and mesic hadrons which are determined by the long-distance features of QCD. Conventional hadrons are made out of constituent quarks, with single gluon exchange being responsible for the binding (perturbative approach). Gluonic hadrons fall into two categories: glueballs and hybrids. Glueballs are predominantly excited states of glue while hybrids are resonances consisting largely of a quark, an antiquark, and excited 




Fig. 7. Charmonium spectrum from LQCD. See [17] for details.

glue. Mesic hadrons are dimesons and/or tetra-quarks, often referred to as multi-quarks or baryonium. They may be viewed as a loosely bound meson-anti-meson system or a diquark-antidiquark ensemble.

The search for glueballs and hybrids has so far mainly been restricted to the mass region below $2.2 \mathrm{GeV} / \mathrm{c}^{2}$. Experimentally, it would be very rewarding to go to higher masses because of the unavoidable problems due to the high density of normal $q \bar{q}$ mesons below $2.5 \mathrm{GeV} / c^{2}$.

The glue tube between quarks (often referred to as f uxtube) is a manifestation of the non-perturbative character of the interaction. It adds degrees of freedom which may manifest in vibrations of the tube. These objects are called hybrids since they carry mesonic and gluonic aspects as non-twobody system. The higher the excitation the more units of angular momentum are carried. These different levels of excitation can be translated into different potentials, one for each mode. Fig. 8 shows this together with the corresponding wave functions.

The additional degrees of freedom (e.g. the vibration of the glue tube) manifest themselves also in a contribution to the quantum numbers of the topology. In the simplest scenario this corresponds to adding the quantum numbers of a gluon $\left(J^{P}=1^{+}\right.$or $1^{-}$depending on if it is a colour-electric or colour-magnetic excitation) to a simple $q \bar{q}$ pair. Therefore they are often referred to as hybrids. This procedure creates, e.g. for $\mathrm{S}$-wave mesons, 8 lowest lying hybrid states. An important experimental aspect here is that 3 out of these 8 states exhibit quantum numbers which cannot be formed by a normal $q \bar{q}$ pair. Therefore these quantum numbers are called exotic. Exotic charmonia are expected to exist in the $3-5 \mathrm{GeV} / \mathrm{c}^{2}$ mass region where they could be resolved and identif ed unambiguously.

All models agree that the lightest exotic state would be $1^{-+}$. Predictions from LQCD are around $4.3 \mathrm{GeV} / c^{2}$. In addition to the lightest exotic state there are seven other hidden charmed hybrids to be discovered. The well accepted picture is that the quartet $1^{--},(0,1,2)^{-+}$is lower in mass than $1^{++},(0,1,2)^{+-}$. The expected splitting is about $100-250 \mathrm{MeV}$ from $1^{-+}$to $0^{+-}$[19]. In addition there is f ne-splitting within the hybrid triplets, so that the levels
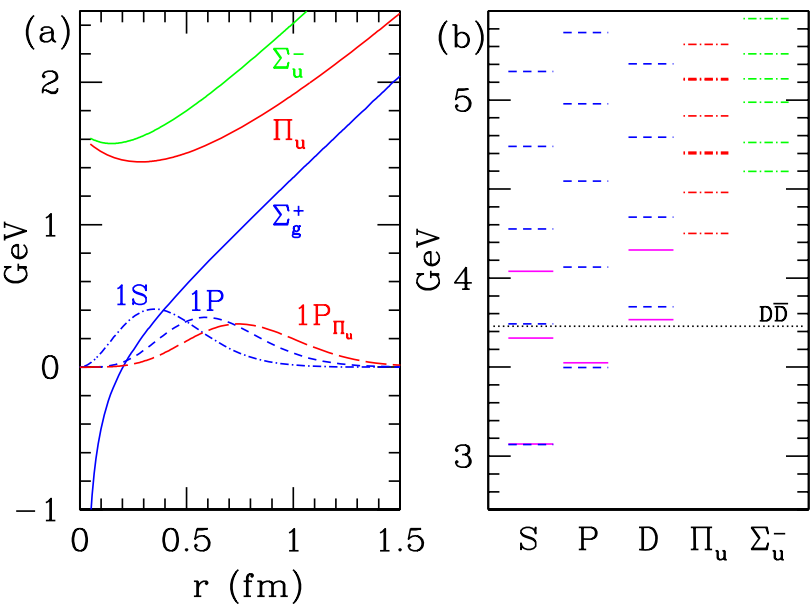

Fig. 8. (a) Heavy quarkonium potentials and wave functions for different excitation levels from LQCD. $\Sigma$ denotes normal one gluon exchange while the excited $\Pi$ potentials are the lowest lying hybrid potentials. In that case, the attraction is not mediated by a single gluon but a string of gluons which carry angular momentum. (b) shows the charmonium spectrum from LQCD. The conventional charmonium states are on the right while the hybrids are found in column $\Pi_{u}$ and $\Sigma_{u}^{-}$. See [18] for details.

are spread over a few hundred $\mathrm{MeV}\left(\right.$ e.g. $4.14 \mathrm{GeV} / c^{2}$ for $0^{-+}$and $4.52 \mathrm{GeV} / \mathrm{c}^{2}$ for $2^{-+}$) [20] which was verif ed by lattice QCD [21]. The actual signature is therefore not just an individual state, but also the pattern of the states.

From experiments at LEAR we know that production rates of such $q \bar{q}$ states are similar to those of states with exotic quantum numbers. Thus, we estimate that the cross sections for the formation and production of charmonium hybrids will be similar to those of normal charmonium states which is in the order of $1 \mathrm{nb}$.

\subsection{Non-perturbative QCD dynamics}

In the quark picture hyperon pair production either involves the creation of a quark-antiquark pair or the knock out of such pairs out of the nucleon sea. Hence, the creation mechanism of quark-antiquark pairs and their arrangement to hadrons can be studied by measuring the reactions of the type $\overline{\mathrm{p}} \mathrm{p} \rightarrow \bar{Y} Y$, where $Y$ denotes a hyperon. By comparing several reactions involving different quark $f$ avours the OZI rule, and its possible violation, can be tested for different levels of disconnected quark-line diagrams separately.

An effective description of reactions in hadron physics relies on the identif cation of the relevant degrees of freedom. At highest energies quark and gluon degrees of freedom seem to describe the observed reactions very accurately. The energy regime for $\bar{p} p$ collisions at HESR is well suited to study the onset of hadron degrees of freedom. In this regime both ansätze are viable. Thus they can be experimentally tested separately and compared to each other.

The parity violating weak decay of most ground state hyperons introduces an asymmetry in the distribution of the decay particles. This is quantif ed by the decay asymmetry parameter and gives access to spin degrees of free- 
dom for these processes, both to the antihyperon/hyperon polarisation and spin correlations. One open question is how these observables relate to the underlying degrees of freedom.

All strange hyperons, as well as single charmed hyperons are energetically accessible in $\bar{p}$ p collisions at HESR. A systematic investigation of these reactions will bring new information on single and multiple strangeness production and its dependence on spin observables. This is particularly true above $2 \mathrm{GeV} / c$ where practically nothing is known about the differential distributions and spin observables. The large amount of observables accessible and the high statistics $\bar{P}$ ANDA data will allow for a partial wave analysis. Thus it will be possible to pin down relevant quantum numbers, coupling constants and possibly fnd new resonances.

As seen from Fig. 9 any measurement of hyperon pair production in the $\overline{\mathrm{P}}$ ANDA energy regime will signif cantly improve the data set. Even in $\Lambda \bar{\Lambda}$ production, where a large data set exists, data points at momenta above $2 \mathrm{GeV} / c$ and a cross check at low momenta would help understanding the production mechanism. For all other hyperon pairs $\bar{P}$ ANDA will provide the frst conclusive insights on the behaviour of the total cross section and frst differential cross sections. The very f rst measurements can be provided for the pair production of charmed hyperons.

Spin observables for the $\bar{\Xi} \Xi$ reaction can be extracted similarly to the $\Lambda \bar{\Lambda}$ case. This will allow for detailed comparisons between the $s \bar{s}$ and $s \bar{s} s \bar{s}$ creation processes. A comparison between the $\Lambda \bar{\Lambda}$ channel, which flters isospin $I=0$, to the $\bar{\Lambda} \Sigma^{0}$ channel (including its charge conjugate channel), which forces $I=1$, gives opportunities to study the isospin dependence of strangeness production. In the naive quark model the spin of the $\Sigma^{0}$ is opposite to that of its constituent strange quark. This should lead to differences in the spin correlations if they are related to the spin state of the created $s \bar{s}$-pair. Studies on the production of charmed hyperons will allow for detailed comparisons between the $\bar{c} c$ and the $s \bar{s}$ creation processes. This may help to disentangle the perturbative contributions from the nonperturbative ones, as the charm production will be mainly probing the hard processes while the strangeness production will be inf uenced by non-perturbative effects.

\subsection{Hadrons in Nuclear Matter}

The study of hadron properties at f nite nuclear densities has a long history. As the most fundamental aspect, the change of hadron masses inside the nuclear medium has been proposed to ref ect a modif cation of the chiral symmetry breaking pattern of QCD due to the f nite density, and thus to be an indicator of changes of quark condensates. In particular, attractive mass shifts ref ecting the reduced quark pair condensate at f nite density have been predicted for vector mesons $[22,23]$. The most signif cant medium effects are thus expected for hadrons which are at rest or have a small momentum relative to the nuclear environment, whereas the non-observation of mass shifts at high momenta not necessarily implies the absence of nuclear potentials. For experimental studies of hadron inmedium properties therefore appropriate conditions of the reaction kinematics have to be selected. Due to the released annihilation energy of almost $2 \mathrm{GeV}$ hadrons produced in antiproton-nucleon collisions may be implanted in the nuclear environment at much lower momenta than e.g. in proton induced reactions.

Besides the mass shift also the change of the width of hadrons inside the nuclear medium is an important observable. In general the width will increase due to the opening of decay channels which are not accessible in the vacuum. The measurement of the modif cation of a hadron's width in the nuclear medium therefore yields information on its inelastic interactions, which is otherwise very difficult to access for unstable hadrons. In the case of very short-lived mesons decaying inside the nucleus, the in-medium width may be measured directly. As an alternative approach, the measurement of the transparency ratio determined from the production cross section with different target nuclei allows to deduce the in-medium width of mesons.

For some observables, the effect of the nuclear medium becomes surprisingly small: this is the Colour transparency phenomenon which is related to the gauge nature of strong interactions. The study of exclusive hard reactions in a nuclear medium constitutes a way to test the factorisation of a short distance process occurring between colour neutral objects whose transverse size is controlled by the hardness of the collision (measured by the momentum transfer $t$ ). Moreover the nuclear medium may even be able to flter out the non short distance dominated part of the amplitude.

The energy range of the HESR and the detection capabilities of the $\bar{P}$ ANDA detector in principle allow to extend studies of the in-medium properties of hadrons into the charm sector by using antiproton-nucleus collisions as entrance channel.

Apart from the determination of nuclear potentials of charmed hadrons, specif c well-def ned problems exist to which the study of antiproton-nucleus collisions with $\bar{P}$ ANDA can contribute valuable information. As an important issue in this respect we see the still open question of the $J / \psi N$ dissociation cross section. This cross section is as yet experimentally unknown, except for indirect information deduced from high-energy $J / \psi$ production from nuclear targets.

Apart from being a quantity of its own interest, the $J / \psi N$ dissociation cross section is closely related to the attempt of identifying quark-gluon plasma (QGP) formation in ultra-relativistic nucleus-nucleus collisions. A signif cant additional, so-called anomalous suppression of the $J / \psi$ yield in high-energy nucleus-nucleus collisions had been predicted due to colour screening of $c \bar{c}$ pairs in a QGP environment [24]. In fact, the CERN-SPS experiments have observed a $J / \psi$ suppression effect increasing with the size of the interacting nuclear system, and interpreted this as signature for QGP formation. The validity of such an interpretation is however based on the knowledge of the "normal" suppression effect due to $J / \psi$ dissociation in a hadronic environment. 
$19^{\text {th }}$ International IUPAP Conference on Few-Body Problems in Physics
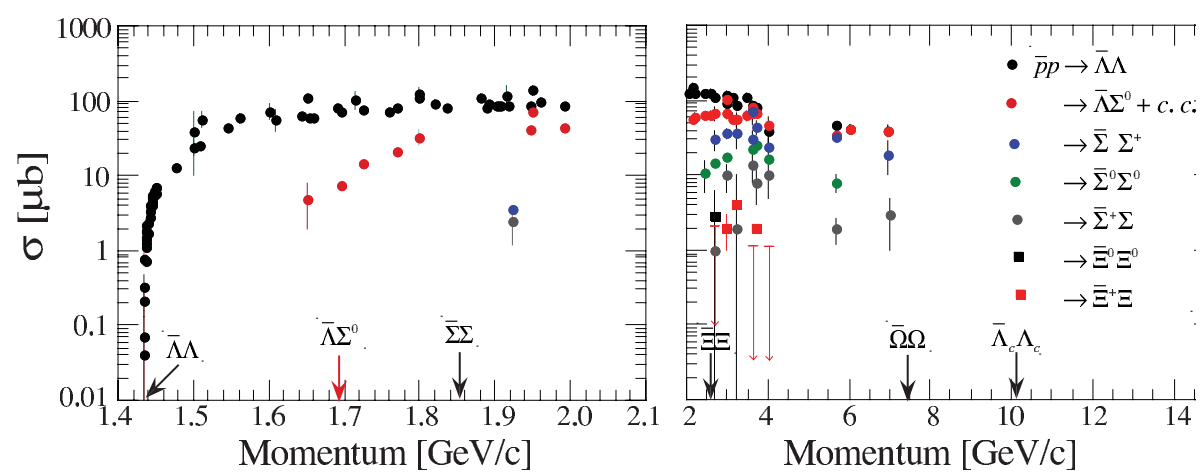

Fig. 9. Total cross sections for the $\overline{\mathrm{p}} \mathrm{p} \rightarrow \bar{Y} Y$ reaction in the momentum range of the HESR. The f gure to the left is an expanded view of the threshold region, which reveals channels of single strangeness production. The f gure to the right shows the experimental situation for momenta above $2 \mathrm{GeV} / c$. The arrows pointing to the momentum axis indicate the threshold momenta for the different hyperon families.

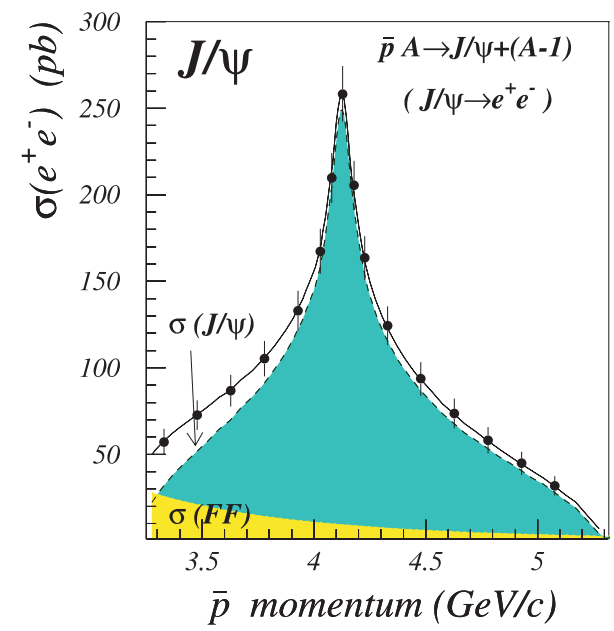

Fig. 10. Simulated cross section for resonant $J / \psi$ production on nuclear protons with internal Fermi momentum distribution as a function of the antiproton momentum [25].

In antiproton-nucleus collisions the $J / \psi N$ dissociation cross section can be determined for momenta around $4 \mathrm{GeV} / c$ with very little model dependence, in contrast to its values deduced from the previous studies. The $J / \psi$ momentum inside the nuclear medium is constrained by the condition that the $\overline{\mathrm{p}} \mathrm{p} \rightarrow J / \psi$ formation proceeds 'on resonance' with a target proton $\left(p_{\overline{\mathrm{p}}}=4.1 \mathrm{GeV} / c\right)$.

In a second step these studies may be extended to higher charmonium states like the $\psi(2 S)$ which requires $\overline{\mathrm{p}}$ momenta around $6.2 \mathrm{GeV} / \mathrm{c}$ for resonant production. This would also allow to determine the cross section for the inelastic process $\psi(2 S) N \rightarrow J / \psi N$ [26] which is also relevant for the interpretation of the ultra-relativistic heavy ion data.

\subsection{Hypernuclear Physics}

Hypernuclei are systems in which up or down quarks are replaced by strange quarks. In this way a new quantum number, strangeness, is introduced into the nucleus. Although single and double $\Lambda$-hypernuclei were discovered many decades ago, only 6 double $\Lambda$-hypernuclei are

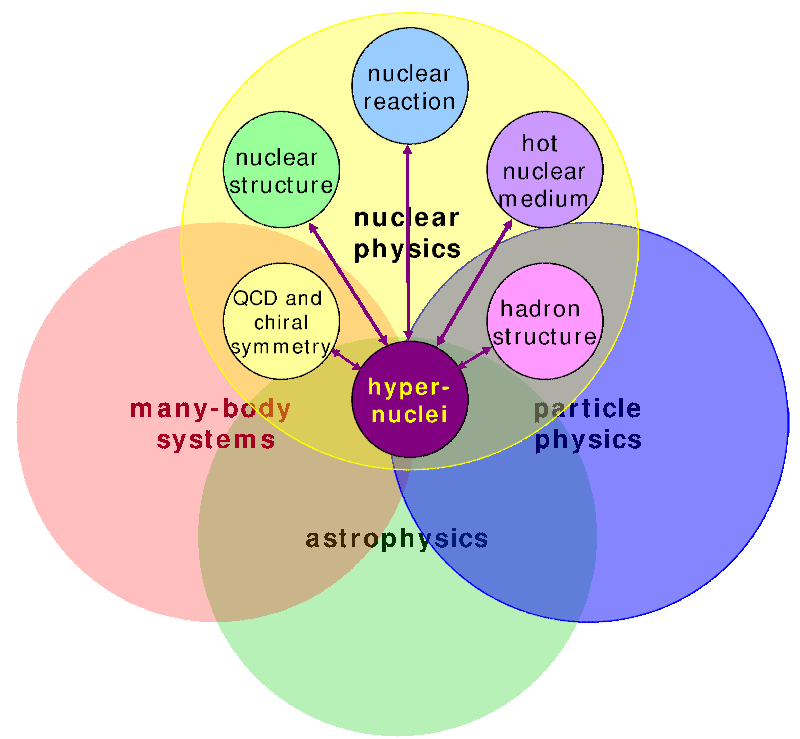

Fig. 11. Hypernuclei and their link to other f elds of physics.

presently known. The availability of antiproton beams at FAIR will allow efficient production of hypernuclei with more than one strange hadron, making PANDA competitive with planned dedicated facilities. This will open new perspectives for nuclear structure spectroscopy and for studying the forces between hyperons and nucleons.

A hyperon bound in a nucleus offers a selective probe of the hadronic many-body problem as it is not restricted by the Pauli principle in populating all possible nuclear states, in contrast to neutrons and protons. On one hand a strange baryon embedded in a nuclear system may serve as a sensitive probe for the nuclear structure and its possible modif cation due to the presence of the hyperon. On the other hand properties of hyperons may change dramatically if implanted inside a nucleus. Therefore a nucleus may serve as a laboratory offering a unique possibility to study basic properties of hyperons and strange exotic objects. Thus hypernuclear physics represents an interdisciplinary science linking many f elds of particle, nuclear and many-body physics (see Fig. 11). 
While it is difficult to study nucleons deeply bound in ordinary nuclei, a $\Lambda$ hyperon not suffering from Pauli blocking can form deeply bound hypernuclear states which are directly accessible in experiments. In turn, the presence of a hyperon inside the nuclear medium may give rise to new nuclear structures which cannot be seen in normal nuclei consisting only of nucleons. Furthermore, a comparison of ordinary nuclei and hypernuclei may reveal new insights in key questions in nuclear physics like for example the origin of the nuclear spin-orbit force [27].

An important goal is to measure the level spectra and decay properties of hypernuclei in order to test the energies and wave functions from microscopic structure models.

While the nucleon-nucleon scattering was extensively studied since the 50's, direct experimental investigations for the YN interactions are still very sparse. Because of their short lifetimes, hyperon targets are not available. Lowmomentum hyperons are very difficult to produce and hyperon-proton scattering is only feasible via the double-scattering technique $[28,29]$. There are only a few hundreds low-momentum $\Lambda$-N and $\Sigma^{ \pm}$-N scattering events available and there is essentially no data on $\Xi-N$ or $\Omega-N$ scattering.

In single hypernuclei the description of hyperons occupying the allowed single-particle states is without the complications encountered in ordinary nuclei, like pairing interactions and so on. The strength of the $\Lambda-N$ strong interaction may be extracted with a description of singleparticle states by rather well known wave functions. Furthermore, the decomposition into the different spin-dependent contributions may be analysed. For these contributions, signif cantly different predictions exist for example from meson exchange-current and quark models.

It is interesting to note that the different $\mathrm{S}=-2$ systems $\Xi^{-}$-atoms and single $\Xi^{-}$-hypernuclei on one side and double $\Lambda \Lambda$ hypernuclei on the other side - provide complementary information on the baryon force: on the one-meson exchange level strange mesons with $\mathrm{I}=1 / 2$ do not contribute to the $\Xi-N$ interaction. At J-PARC f rst precise studies of $\Xi$-atoms are planned [30]. At $\overline{\mathrm{P}} \mathrm{ANDA}$ not only $\Xi^{-}$atoms but also $\Omega^{-}$atoms can be studied for the f rst time thus providing unique information on the nuclear optical potential of $\Omega^{-}$baryons. The $\Omega$ hyperon is particularly interesting because due to it's long lifetime and it's spin of $3 / 2$ it is the only 'elementary' baryon with a non-vanishing spectroscopic quadrupole moment.

In the $\bar{P}$ ANDA experiment, bound states of $\Xi$ hypernuclei will be used as a gateway to form double $\Lambda$ hypernuclei [31]. The production of low momentum $\Xi^{-}$hyperons and their capture in atomic levels is therefore essential for the experiment. At $\overline{\mathrm{P}}$ ANDA the reactions $\bar{p}+p \rightarrow \Xi^{-} \bar{\Xi}^{+}$and $\bar{p}+n \rightarrow \Xi^{-} \bar{\Xi}^{0}$ followed by re-scattering of the $\Xi^{-}$within the primary target nucleus will be employed. After stopping the $\Xi^{-}$in an external secondary target, the formed $\Xi$ hypernuclei will be converted into double $\Lambda$ hypernuclei. This two-step production mechanism requires major additions to the usual simulation package PandaRoot as well as the $\overline{\mathrm{P}}$ ANDA setup. Mandatory for this experiment is a modular and highly fexible setup of the central $\bar{P}$ ANDA detector.

\section{Strong Field Atomic Physics}

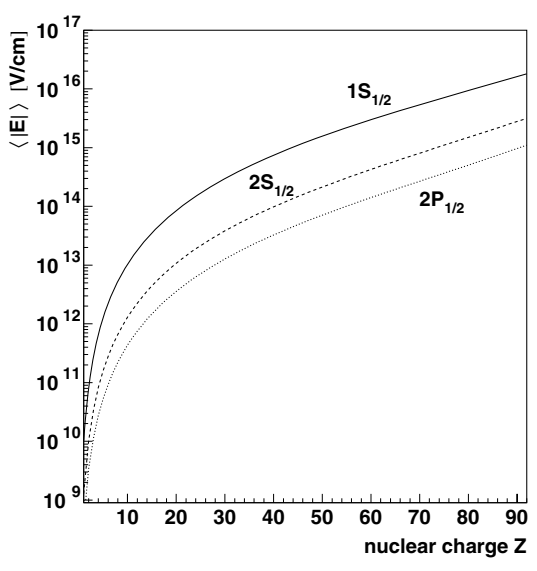

Fig. 12. Expectation value of the electric $f$ eld strength for the lowest-lying states of a hydrogenlike atom in the range $Z=1-$ 92 [34].

The international FAIR Facility for Antiproton and Ion Research has key features that offer a wide range of new and challenging opportunities for atomic physics in the strong feld where the interplay between correlation, relativity and quantum-electrodynamics is of particular interest. Within the SPARC collaboration [32,33] we plan experiments in two major research areas: collision dynamics in strong electromagnetic felds and fundamental interactions between electrons and heavy nuclei up to bare uranium. In this area we will use the relativistic heavy ions for a wide range of collision studies. In the extremely short, relativistically enhanced f eld pulses, the critical f eld limit (Schwinger limit) for lepton pair production can be surpassed by orders of magnitudes and a breakdown of perturbative approximations for pair production is expected. The detection methods of reaction microscopes will give the momentum of all fragments when atoms or molecules are disintegrating in strong f eld pulses of the ions, an ideal tool to study correlated multi-body dynamics. For medium and low energies, the cooler rings NESR - a "second-generation" ESR - and the low-energy ring LSR, with optimized features and novel installations such as an ultra-cold electron target will be exploited for collision studies. Fundamental atomic processes can be investigated in a kinematically complete fashion for the interaction of cooled heavy-ions up to He-, H-like and bare uranium with photons, electrons and atoms. These studies extend into the low-energy regime where the atomic interactions are dominated by strong perturbations but by also correlation and quasi-molecular effects.

The other class of experiments will focus on structure studies of selected highly-charged ion species, a f eld which is still largely unexplored. The properties of stable and unstable nuclei will become accessible by atomic physics techniques along with precision tests of quantum electro- 


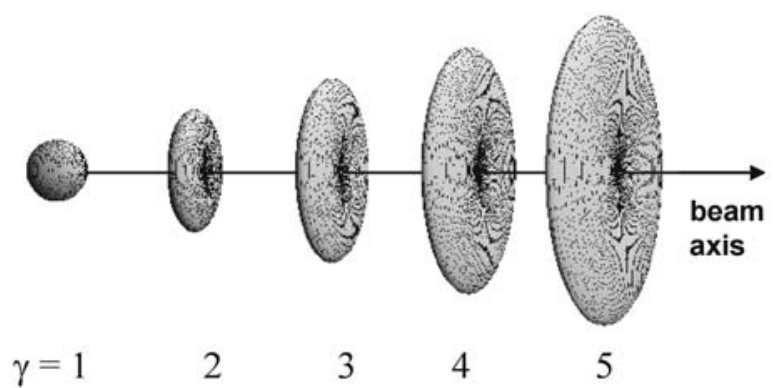

Fig. 13. Angular dependence of the electric radial $\mathrm{f}$ eld strengths of a point charge moving with a Lorentz factor $\gamma(\gamma=1,2,3,4$, and 5) as observed in the laboratory frame [33].

dynamics (QED) in extremely strong electromagnetic $f$ elds (the extreme f eld strength probed for instance by a $1 \mathrm{~s} \mathrm{elec-}$ tron in a H-like heavy ion is exemplif ed in f gure 12). In the strong feld domain, He-like ions, the simplest manyelectron system, are a particular challenge for experiment as well as for theory. Another important scenario for this class of experiments will be the slowing-down, trapping and cooling of particles in the ion trap facility HITRAP located at FLAIR. This may enable not only high-accuracy experiments in the realm of atomic and nuclear physics but as well highly-sensitive tests of the Standard Model.

In the following a few particular examples of the planned experimental activities the SPARC collaboration will be discussed whereby focussing on the high energy aspect.

\subsection{Quantum Dynamics in Strong Electromagnetic Fields}

The transverse electric and magnetic components of the electromagnetic felds associated with the moving ions steadily increase with $\gamma$ and become almost equal in magnitude while the duration of the electromagnetic pulse decreases (see Fig. 4.1) [35]. Therefore in collisions of such ions with atoms, the atom experience a huge f eld strength which is comparable to the felds of the most intense lasers currently available, however on a much shorter time scales (sub-attoseconds). A high-energy $(\gamma>2)$ storage ring brings us in a position to extend the frontiers of our present knowledge in atomic physics into the regime of extreme conditions perfectly suited of a test of nonperturbative QED. While at the most fundamental level a rigorous understanding of nonperturbative QED is essential for the scientif $\mathrm{c}$ progress in atomic physics of strong felds, there are major challenges in the feld of highly relativistic atomic collisions. In particular, as a result of the large electromagnetic $\mathrm{f}$ elds associated with heavy ions in relativistic motion, pair production becomes a very important reaction channel.

Explicit calculations for uranium on uranium collisions at $10 \mathrm{GeV} / \mathrm{u}(\gamma=10)[36]$ cross-sections of close to $10 \mathrm{barn}$ have been predicted. We note, that for such collision parameters (as covered by the new FAIR facility $(2<\gamma<$

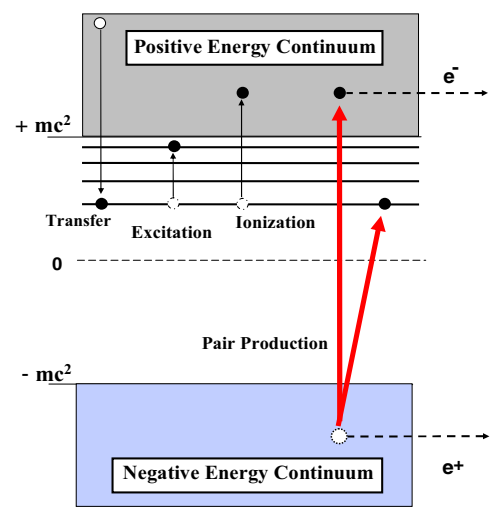

Fig. 14. Energy diagram of the single-particle Dirac equation and basic atomic processes which occur in ion-atom collisions.

35)) a nonperturbative treatment is compulsory. Also we note that at extreme energies, in the region of hundreds of $\mathrm{GeV} / \mathrm{u}$, there is good agreement between theory and experiment. However, the situation is much less satisfactory at intermediate relativistic energies of a few $\mathrm{GeV} / \mathrm{u}$. Here, even the target charge dependence is not well understood $[35,37]$. There are very few and only inclusive measurements of pair production available in the intermediate relativistic regime, the new facility would be the only one capable worldwide of $f$ lling this important gap. In particular, differential measurements as function of the impact parameter are urgently needed since they are expected to display the nonperturbative dynamical structure of the QED vacuum. This is especially true for bound-free pair creation where at small impact parameters the strong electromagnetic $f$ eld can lead to an enhanced production probability.

In addition, these high-energy collisions may enable an exploration of novel electromagnetic phenomena associated with dynamically induced correlated many-electron processes such as multiple ionization/excitation, the innershell vacancy production with simultaneous pair production or the production of multiple $e^{+}-e^{-}$pairs. One may also mention the search for recombination followed by $e^{+}-$ $e^{-}$pair production instead of photon emission. This higherorder process, presumably requires high collision energies, is similar to di-electronic recombination, but with an electron being excited from the negative to the positive continuum. All these processes can be studied at an experimental area behind the SIS100/300 in single-pass and f xed-target experiments at $\gamma$ values up to $\gamma=10$.

\subsection{Laser Spectroscopy}

A typical application of precision laser spectroscopy will be the excitation of transitions starting from the ground state of highly charged, e.g. lithium-like ions. At the NESR, photon energies of around $110 \mathrm{eV}$ in the laboratory frame would be sufficient to induce the $280 \mathrm{eV} 2 \mathrm{~s}_{1 / 2}-2 \mathrm{p}_{1 / 2}$ transition in uranium when the laser light is counter-propagating 
to the ions [38]. Such a source could be provided by an Xray laser pumped by multi-terawatt pulses from the $10 \mathrm{~J}$ front-end of the laser system PHELIX available at GSI [40]. The experimental procedure will be similar to the established scheme used for laser spectroscopy of the groundstate hyperf ne splitting in hydrogen-like ions. Light emitted after laser excitation is detected in the forward direction. This leads to an additional Doppler shift into an energy range between two and three times the laser energy. These energetic photons can be efficiently and selectively detected. Another application will be the study of lowlying metastable states in moderately charged heavy ions. In these cases many-electron correlation effects can be studied in great detail. Alternatively, exploiting the higher energies available in the SIS300, even photon energies in the visible range will be sufficient for excitation of the $2 \mathrm{~s}_{1 / 2}$ - $2 p_{1 / 2}$ transition in lithium-like uranium. For such ions, a beam energy of $21.3 \mathrm{GeV} / \mathrm{u}$ corresponding to a Lorentz factor of $\gamma=35$ can be reached at SIS300. Since after laser excitation the emitted photon again will be strongly Doppler shifted by $2 \times \gamma$, high detection sensitivity and good suppression of scattered laser light can be reached. Also, f xed-frequency lasers can be used because they can be tuned via the Doppler shift.

The experimental setup consists of the combination of two very precise experimental methods which are based on a laser and a x-ray crystal spectrometer. The $2 \mathrm{~s}_{1 / 2}-2 \mathrm{p}_{1 / 2}$ transition can be excited for all elements up to uranium with a pulsed frequency-doubled laser beam. For the case of lithium-like uranium, a laser photon energy $\hbar \omega_{L}=5.87 \mathrm{eV}$ is shifted in the moving system to $\hbar \omega_{0}=280.6 \mathrm{eV}$ which is the $2 s_{1 / 2}-2 p_{1 / 2}$ transition energy. The emitted fuorescence photon is boosted into the hard $\mathrm{x}$-ray region and appears in the laboratory system in the forward direction at $\hbar \omega_{x}=13.4 \mathrm{keV}$.

It has been pointed out [38] that with currently existing laser systems with a relative line width of $5 \cdot 10^{-7}$ and a mean power of $5 \mathrm{~W}$ at a repetition rate of $10 \mathrm{kHz}$, shining on a collection of $10^{6}$ lithium-like ions circulating as a bunch with an energy spread of $10^{-4}$ and observed by an $\mathrm{x}-$ ray monochromator with a resolution of $5.7 \cdot 10^{-5}$, the transition energy of $280 \mathrm{eV}$ in uranium can be measured with an accuracy of $0.007 \mathrm{eV}$ within one hour. Compared with the currently best value of 280.645(15) eV [39], this will lead to a signif cant increase in precision and will provide a highly sensitive test of QED and relativistic-correlation calculations for three-electron systems.

\subsection{Cooling and Polarization of Ions by Laser Light}

Ions stored in rings or traps can also be cooled by interaction with laser photons. This process is possible using the high interaction cross section at the resonance of ionic transitions.

The possibility of increasing the beam quality of stored ions by laser cooling has been proven for highly charged ions recently at the ESR storage ring. The high velocity in the SIS100/SIS300 will make this method applicable to a wide range of low- and highly-charged ions, even using conventional laser sources. Laser cooling can therefore produce a variety of highly relativistic beams of highest quality even without the necessity of electron cooling. A very attractive feature will be the bunch contraction of cooled ions which has also been observed at smaller storage rings. This results in an increase of the pulse intensity of ion bunches which is of particular relevance for applications in plasma physics. By using a conventional laser system, laser cooling requires a time of about $10 \mathrm{~s}$ with, depending mainly on the radiative lifetime of the transition used. In some favorable cases, much faster cooling can be expected. With circularly polarized laser beams the nuclei can also be polarized. If the polarization can be maintained in the ring or at least in some part of it, such polarized and cooled beams may open up a variety of novel experiments related to fundamental physics [43].

Here we like to comment on a novel experimental possibility to detect the orientation of the polarization plane of projectile $\mathrm{x}$-rays emitted in relativistic ion-atom collisions. This technique is of great importance for experiments aiming at the control of the spin-polarization of stored particles. As examples we like to mention various experiments for the test of the standard model via the search of an electric dipole-moment of elementary particles as well as the study of parity violation effects in simple atomic systems at high-Z $[41,1]$. Recently, detailed theoretical investigations predicted that the process of radiative recombination transitions [42-44] may reveal a possible spin polarization of the particles involved in the interaction (electrons or ions). Here, the spin polarization of the particles leads to a rotation of the photon polarization vector out of the scattering plane. Experimentally, this topic can now be addressed with high efficiency by a new generation of solid state detectors which exploit Compton scattering inside the detector for an accurate polarization determination. These Compton polarimeters, developed for the SPARC collaboration, are capable to provide energy as well as position information along with real multi-hit capability [45-47].

\subsection{Dielectronic Recombination, a Tool to Determine Nuclear Charge Radii}

Dielectronic recombination (DR) of ions, having at least one electron, with free electrons turned out to be a novel and sensitive tool to study the interplay between the effects of correlation and QED for the atomic structure at high-Z [48]. The possibility of producing highly-charged heavy ions and of storing them as low-emittance ion beams in a storage ring opened a new window for the investigation of the recombination of ions and electrons at low relative velocities. In this resonant process a free electron is captured and the excess energy excites one of the bound electrons to a higher state. This is the time-reversed Auger effect.

The NESR with a second independent electron target will allow for the f rst time the study of the electron-electron interaction also for the innermost bound electrons in very heavy systems. At the ESR, having no second electron target, such studies were not possible up to now. An indepen- 


\section{$19^{\text {th }}$ International IUPAP Conference on Few-Body Problems in Physics}

dent electron target at the NESR will allow also to improve the precision and accuracy of the measurements since the ion beam can be cooled permanently during the DR experiments.

Dielectronic recombination also allows studies of nuclear charge radii. This method is applicable to radioactive isotopes with a half-life longer than about $1 \mathrm{~min}$. If the free electron is captured into a low-lying bound state, the comparison of precise measurements with theory can be used as a novel tool to determine nuclear radii. For the particular case of ${ }^{A} \mathrm{Nd}^{57}$, the isotope shift of the DR resonances have very recently been studied in an pilot experiment at the ESR, demonstrating the potential of this technique [49].

\section{Summary}

During the next years the Facility for Antiproton and Ion research FAIR will be constructed in Darmstadt. Once this facility is operational, it will provide for the exploration of the scientif c terra incognita in many research felds. The current manuscript discusses a variety of possibilities in few-body research which FAIR offers in hadron, nuclear and atomic physics. We showed that FAIR will allow to map out the driplines up to medium-mass nuclei and to explore the structure of nuclear states close and in the continuum. The hadron program around the PANDA detector plans to generate QCD states in antiproton annihilation reactions in order to shed more light on the complex structure of hadrons and the understanding of binding among quarks. The atomic physics program at FAIR can study correlations among electrons in helium- or-lithiumlike heavy ions like uranium and will perform fundamental tests of QED.

The FAIR research program at a whole will also open up a new era in nuclear astrophysics as many of the research topics are intimately related to fore-front research in astrophysics. For example, the exotic nuclei, which are available at FAIR as secondary beams with unmatched intensities, are crucial for the understanding of the dynamics of astrophysical objects like core-collapse supernovae, the origin of the elements in the Universe and also the structure of compact objects like neutron stars. Further improvement here will also come from the study of hypernuclei performed at the $\bar{P}$ ANDA detector which will advance our knowledge of the interaction among hyperons and hence the Equation of State at densities expected to exist inside of neutron stars. Neutron stars are also known to have extreme electric surface felds. The correlations of electrons under such conditions can be investigated by the atomic physics program at FAIR. Of course, also the plasma physics program at FAIR relates to astrophysical objects like the interior of the Sun or of gas planets like Jupiter.

Thus it is obvious that more than 2500 scientists worldwide are eagerly preparing their experiments to be ready once FAIR is operational.

\section{Acknowledgement}

The support by the ExtreMe Matter Institute EMMI in the framework of the Helmholtz Alliance HA216/EMMI is acknowledged. KL thanks support of the Deutsche Forschungsgemeinschaft under contract No. SFB 634.

\section{References}

1. W.F. Henning et al., An International Accelerator Facility for Beams of Ions and Antiprotons, GSI Darmstadt 2001, http://www.gsi.de/GSI Future/cdr/

2. http://www.gsi.de/fair/experiments/CBM

3. http://www.gsi.de/fair/experiments/NUSTAR

4. H.-Th. Janka et al., Phys. Rep. 442 (2007) 38

5. W. Erni et al., Physics Performance Report for PANDA, GSI Darmstadt 2009, http://wwwpanda.gsi.de/archive/public/panda_pb.pdf, Future/cdr/arXiv:0903.3905v1

6. https://www.gsi.de/forschung/phelix/Experiments/ FAIR/WDM

7. M.V. Zhukov, L.V. Chulkov, B.V. Danilin and A.A. Korsheninnikov, Nucl. Phys. A533 (1991) 428.

8. A. Cobis, D. Fedorov and A. Jensen, Phys. Rev. Lett. 79 (1997) 2411.

9. B. Danilin, I. Thompson, J. Vaagen and M. Zhukov, Nucl. Phys. A632 (1998) 383.

10. T. Aumann et al., Phys. Rev. C 59 (1999) 1252.

11. M. Meister et al., Nucl. Phys. A 723 (2003) 13.

12. N.B. Shulgina, B.V. Danilin, L.V. Grigorenko, M.V. Zhukov, J.M. Bang, Phys. Rev. C 62 (2000) 014312.

13. M. Meister et al., Phys. Rev. Lett. 91 (2003) 162504.

14. Y u. Aksyutina et al., Phys. Lett. B 679 (2009) 191.

15. Y u. Aksyutina et al., Phys. Lett. B 666 (2008) 430.

16. L. Chulkov et al., Nucl. Phys. A 759 (2005) 43.

17. Brambilla, N. et al., Quarkonium Working Group: Heavy quarkonium physics, arXiv hep-ph/0412158

18. K.J. Juge, J. Kuti and C. Morningstar, Phys. Rev. Lett. 90 (2003) 161601

19. T. Manke et al., Phys. Rev. D 57 (1998) 3829

20. J. Merlin and J. Paton, Phys. Rev. D 35 (1987) 1668

21. K.J. Juge, J. Kuti and C.J. Morningstar, Nucl. Phys. (Proc. Suppl.) B 86 (2000) 397

22. G.E. Brown and M. Rho, Phys. Rev. Lett. 66 (1991) 2720

23. T. Hatsuda and S.H. Lee, Phys. Rev. C 46 (1992) 34

24. T. Matsui and H. Satz, Phys. Lett. B 178 (1986) 416

25. K. Seth, Proc. Int. Workshop on the Structure of Hadrons, Hischegg, Austria, 2001,

26. L. Gerland, L. Frankfurt and M. Strikman, Phys. Lett. B 619 (2005) 95

27. N. Kaiser and W. Weise, Phys. Rev. C 71 (2005) 015203

28. Y. Kondo et al., Nucl. Phys. A 676 (2000) 371

29. J.K. Ahn et al., Phys. Lett. B 633 (2006) 214

30. K. Tanida et al., Proc. of the IX International Conference on Hypernuclear and Strange Particle Physics (2007) 145, SIF and Springer-Verlag Berlin Heidelberg 
31. J. Pochodzalla, Nucl. Phys. A 754 (2005) 430c

32. http://www.gsi.de/ sparc/

33. Th. Stöhlker et al., Nucl. Instr. Meth. B, 205 (2003) 156.

34. G. Soff et al., Adv. Quant Chem. 20, (1998) 125

35. J. Eichler, W.E. Meyerhof Relativistic Atomic Collisions (Academic Press, San Diego, 1995)

36. M. Gail, N. Grün, and W. Scheid, J. Phys. B 31, 4645 (1998).

37. A. Belkacem et al. PRA56, (1997) 2806

38. H. Backe, GSI Workshop on its Future Facility (October 18-20, 2000)

39. P. Beiersdorfer et al., Phys. Rev. Lett. 95, 233003 (2005).

40. V. Bagnoud et al., Applied Physics B: DOI 10.1007/s00340-009-3855-7, (2010)

41. L. N. Labzowsky et al., Phys. Rev. A 63, (2001) 054105

42. J. Eichler and Th. Stöhlker, Physics Reports 429, (2007) 1

43. A. Surzhykov et al., Phys. Rev. Lett. 88, (2002) 153001

44. S. Tashenov et al., Phys. Rev. Lett. 97, (2006) 223202

45. U. Spillmann et al. Rev. Sci. Instr. 79, (2008) 083101

46. S. Hess, PhD Thesis University of Frankfurt (2010); S. Hess et al. to be submitted to Phys. Rev. Lett.

47. Th. Stöhlker et al., European Physical Journal-Special Topics 169, (2009) 5

48. C. Brandau et al., , Physica Scripta T80B, (1999) 318

49. C. Brandau et al., Phys. Rev. Lett. 100, 073201 (2008) 